\title{
Plasma levels of lipometabolism-related miR-122 and miR-370 are increased in patients with hyperlipidemia and associated with coronary artery disease
}

Wei Gao ${ }^{1+}$, Hui-Wei He ${ }^{2 \dagger}$, Ze-Mu Wang ${ }^{1}$, Huan Zhao ${ }^{1}$, Xiao-Qing Lian ${ }^{1}$, Yong-Sheng Wang ${ }^{1}$, Jun Zhu', Jian-Jun Yan ${ }^{1}$, Ding-Guo Zhang ${ }^{1}$, Zhi-Jian Yang ${ }^{1}$ and Lian-Sheng Wang ${ }^{1 *}$

\begin{abstract}
Background: Hyperlipidemia plays a crucial role in the development and progression of coronary artery disease (CAD). Recent studies have identified that microRNAs (miRNAs) are important regulators of lipid metabolism, but little is known about the circulating levels of lipometabolism-related miRNAs and their relationship with the presence of CAD in patients with hyperlipidemia.

Methods: In the present study, we enrolled a total of 255 hyperlipidemia patients with or without CAD and 100 controls with normal blood lipids. The plasma levels of four known lipometabolism-related miRNAs, miR-122, miR-370, miR-33a, and miR-33b were quantified by real-time quantitative PCR. Blood levels of total cholesterol (TC), triglyceride (TG), low density lipoprotein cholesterol (LDL-C), and high density lipoprotein cholesterol were determined. Furthermore, the severity of CAD was assessed with the Gensini score system based on the degree of luminal narrowing and its geographic importance.

Results: Our results revealed for the first time that plasma levels of miR-122 and miR-370 were significantly increased in hyperlipidemia patients compared with controls, and the levels of miR-122 and miR-370 were positively correlated with TC, TG, and LDL-C levels in both hyperlipidemia patients and controls. Multiple logistic regression analysis demonstrated that the increased levels of miR-122 and miR-370 were associated with CAD presence, even after adjustment for other cardiovascular risk factors. Furthermore, miR-122 and miR-370 levels were positively correlated with the severity of CAD quantified by the Gensini score. However, both miR-33a and miR-33b were undetectable in plasma.
\end{abstract}

Conclusions: Our results suggest that increased plasma levels of miR-122 and miR-370 might be associated with the presence as well as the severity of CAD in hyperlipidemia patients.

Keywords: MicroRNA, Hyperlipidemia, Coronary artery disease, Plasma, Gensini score

\footnotetext{
* Correspondence: drlswang@njmu.edu.cn

${ }^{\dagger}$ Equal contributors

'Department of Cardiology, The First Affiliated Hospital of Nanjing Medical University, Nanjing, 210029, China

Full list of author information is available at the end of the article
} 


\section{Background}

Atherosclerosis, the major cause of CAD, is characterized by accumulation of lipid in the arterial wall resulting in narrowing of the vessel lumen [1]. Disorders of lipid homeostasis have been confirmed to play an important role in the development and progression of $\mathrm{CAD}$, and hyperlipidemia has thus been identified as a major risk factor for CAD [2].

MicroRNAs (miRNAs) are a class of endogenous, noncoding, single-stranded short RNAs, that negatively regulate gene expression at the post-transcriptional level by binding to the 3'untranslated regions of target mRNAs [3]. Recently, accumulating data from in-vivo and in-vitro studies have indicated that a variety of miRNAs, particularly miR-122, miR-370, and miR-33a/b, play crucial roles in lipid metabolism [4]. Several key genes involved in fatty acid synthesis and oxidation were found to be regulated by miR-122, and silencing of miR-122 resulted in decreased plasma levels of both total cholesterol (TC) and triglyceride (TG) [5-8]. Another miRNA, miR-370, was found to have similar effects on lipid metabolism as miR-122. In addition, miR-370 could directly down-regulate the expression of carnitine palmitoyl transferase $1 \alpha$ gene, which controls fatty acid oxidation [9]. The miR-33 family, containing two isoforms (miR-33a and miR-33b), was also shown to target multiple lipid metabolism-associated genes [1014]. Inhibition of miR-33 elevated plasma high density lipoprotein cholesterol (HDL-C) levels in both normal and high-fat-diet-fed mice [10-12].

To date, miRNAs in plasma and serum have been considered as promising novel biomarkers for diagnosis and prognosis of cardiovascular diseases, especially CAD $[15,16]$. However, the circulating levels of lipometabolism-related miRNAs and their relationship with the presence of CAD in patients with hyperlipidemia remain to be determined. In the present study, we aimed to identify the plasma levels of four most prominent lipometabolism-related miRNAs, miR-122, miR-370, miR-33a, and miR-33b in patients with hyperlipidemia, as well as to determine the relationship between the miRNAs levels and the presence of CAD.

\section{Materials and Methods \\ Study subjects}

A total of 355 patients who underwent coronary angiography for the diagnosis and interventional treatment of CAD were recruited from inpatients admitted to the First Affiliated Hospital of Nanjing Medical University. Hyperlipidemia was defined as TC level of $\geq 5.72 \mathrm{mmol} / \mathrm{L}$ and/or TG level of $\geq 1.70 \mathrm{mmol} / \mathrm{L}$, or if the patient was being treated with lipid-lowering medication [17]. Diagnosis of CAD was confirmed by coronary angiography performed with the Judkins technique using a quantitative coronary angiographic system [18]. CAD was defined as angiographic evidence of at least one segment of a main coronary with more than $50 \%$ luminal narrowing. CAD patients were divided into single-, double-, and triple-vessel disease subgroups according to the number of significantly stenosed vessels with reference to the Coronary Artery Surgery Study classification. The severity of CAD was assessed with the Gensini score system based on the degree of luminal narrowing and its geographic importance [19]. Two cardiologists who were unaware of the patients included in this study assessed the angiograms. Patients with histories of significant concomitant diseases including hepatic failure, renal failure, hepatitis, cardiomyopathy, congenital heart disease, bleeding disorders, previous thoracic irradiation therapy, and malignant diseases were excluded. Subjects without clinical evidence of hyperlipidemia and CAD were randomly recruited as control subjects. Hypertension and diabetes were defined as described in our previous studies [20]. In brief, hypertension was defined as resting systolic blood pressure $\geq 140 \mathrm{mmHg}$ and/or diastolic blood pressure $\geq 90 \mathrm{mmHg}$ or in the presence of active antihypertensive treatment. Diabetes was defined as fasting blood glucose $\geq 7.0 \mathrm{mmol} / \mathrm{L}$ or a diagnosis of diabetes needing diet or anti-diabetic therapy. Individuals who formerly or currently smoked $\geq 10$ cigarettes per day for at least 2 years were defined as smokers. This study was approved by the First Affiliated Hospital Ethics Committee of Nanjing Medical University, and informed consent was obtained from each participant.

\section{Laboratory measurements}

Fasting blood sample was collected from each subject and anticoagulated with ethylenediamine tetraacetic acid (EDTA) dipotassium salt in the early morning. Sample was separated immediately by centrifugation at $3000 \mathrm{~g}$ for $15 \mathrm{~min}$ at $4^{\circ} \mathrm{C}$ to retrieve plasma. The plasma was then stored at $-80^{\circ} \mathrm{C}$ until assayed. The levels of plasma TC, TG, HDL-C, low density lipoprotein cholesterol (LDL-C), and fasting glucose were detected by an automated chemical analyzer (Olympus Automated Chemistry Analyzer AU5400, Japan).

\section{RNA extraction and reverse transcription (RT)}

Total RNA was isolated from $400 \mu \mathrm{L}$ of plasma using the mirVana ${ }^{\mathrm{TM}}$ PARIS $^{\mathrm{TM}}$ Kit (Ambion, Austin, TX) according to the manufacturer's instructions with modification. For normalization of sample-to-sample variation, $25 \mathrm{fmol}$ of synthetic C.elegans miRNA cel-miR-39 (Qiagen, Germany) was added to each sample after addition of $2 \times$ Denaturing Solution (Ambion, Austin, TX) [21]. RNA was dissolved in $100 \mu \mathrm{L}$ of RNase-free water, and then stored at $-80^{\circ} \mathrm{C}$ until analysis. 
Total RNA was reverse transcribed using the TaqMan ${ }^{\circledR}$ MiRNA Reverse Kit (Applied Biosystems, Foster, $\mathrm{CA}$ ) in $15 \mu \mathrm{L} \mathrm{RT}$ reaction containing $5 \mu \mathrm{L}$ of $\mathrm{RNA}$ extract, $0.15 \mu \mathrm{L}$ of $100 \mathrm{mM}$ dNTPs (with dTTP), $1 \mu \mathrm{L}$ of multiscribe reverse transcriptase $(50 \mathrm{U} / \mu \mathrm{L}), 1.5 \mu \mathrm{L}$ of $10 \times$ RT buffer, $0.19 \mu \mathrm{L}$ of RNase inhibitor $(20 \mathrm{U} / \mu \mathrm{L})$, $4.16 \mu \mathrm{L}$ of RNase-free water, and $3 \mu \mathrm{L}$ of $5 \times$ miRNAspecific stem-loop RT primer (Applied Biosystems, Foster, CA). For synthesis of cDNA, the RT reaction was incubated at $16^{\circ} \mathrm{C}$ for $30 \mathrm{~min}$, at $42^{\circ} \mathrm{C}$ for $30 \mathrm{~min}$, at $85^{\circ} \mathrm{C}$ for $5 \mathrm{~min}$, and then held at $4^{\circ} \mathrm{C}$. The cDNA product was stored at $-20^{\circ} \mathrm{C}$ until analysis.

\section{Real-time quantification PCR to detect plasma miRNA levels}

For Real-time quantitative PCR (qRT-PCR), $1.33 \mu \mathrm{L}$ of the cDNA product was used as template in $20 \mu \mathrm{L}$ reaction containing $1 \mu \mathrm{L}$ of TaqMan miRNA Assay, $7.67 \mu \mathrm{L}$ of RNase-free water, and $10 \mu \mathrm{L}$ of TaqMan ${ }^{\circledR} 2 \times$ Universal PCR Master Mix, No AmpErase ${ }^{\circledR}$ UNG (Applied Biosystems, Foster, CA). qRT-PCR was performed with 7900HT real-time PCR system(Applied Biosystems, Foster, CA) at $95^{\circ} \mathrm{C}$ for $10 \mathrm{~min}$, followed by 40 cycles of $95^{\circ}$ $\mathrm{C}$ for $15 \mathrm{~s}$ and $60^{\circ} \mathrm{C}$ for $1 \mathrm{~min}$. Triplicate measurements were obtained for each sample on a 384-well plate. Data were analyzed with SDS Relative Quantification Software version 2.2.2 (Applied Biosystems, Foster, CA), with the automatic $\mathrm{Ct}$ setting for assigning baseline and threshold for $\mathrm{Ct}$ determination. The relative expression level of each individual miRNA after normalization to cel-miR39 was calculated using the $2^{-\nabla \nabla C t}$ method.

\section{Statistical analysis}

Normality of distribution was assessed using the Kolmogorov-Smirnov test. Comparison between 2 groups was performed with Student's t tests or Mann-Whitney U tests. For comparison of more than 2 groups, one-way ANOVA or Kruskal-Wallis test was used as appropriate. Pearson $\chi^{2}$ test was used to compare qualitative variables represented as frequencies. The correlations between plasma levels of miRNAs and other variables were calculated using Spearman correlation coefficient. Univariate analysis and multivariate logistic regression analysis were taken to determine the variables that independently contributed to the presence of CAD. Odds ratio (OR) and $95 \%$ confidence interval $(\mathrm{CI})$ were calculated. All tests were two-sided and $P<0.05$ was considered statistically significant. Statistical analyses were performed using PASW 18.0 (IBM SPSS, Inc., Chicago, USA).

\section{Results}

\section{Characteristics of study subjects}

The clinical characteristics of the sample population are summarized in Table 1. Compared with the controls, patients with hyperlipidemia had higher levels of TC, TG, LDL-C, but lower HDL-C. However, no significant

Table 1 Characteristics of study subjects

\begin{tabular}{|c|c|c|c|c|c|c|}
\hline Characteristic & $\begin{array}{l}\text { Control } \\
(n=100)\end{array}$ & $\begin{array}{l}\text { All } \\
\text { Hyperlipidemia } \\
(n=255)\end{array}$ & $\begin{array}{l}P \\
\text { value }\end{array}$ & $\begin{array}{l}\text { Hyperlipidemia } \\
\text { without CAD } \\
(n=100)\end{array}$ & $\begin{array}{l}\text { Hyperlipidemia } \\
\text { with CAD } \\
(n=155)\end{array}$ & $\begin{array}{l}P \\
\text { value }\end{array}$ \\
\hline Age (years) & $63.0 \pm 10.7$ & $65.2 \pm 10.7$ & 0.162 & $65.2 \pm 10.2$ & $65.3 \pm 11.0$ & 0.850 \\
\hline Male, n (\%) & $35(70.0 \%)$ & $162(63.5 \%)$ & 0.382 & 57 (57.0 \%) & $105(67.7 \%)$ & 0.082 \\
\hline BMI $\left(\mathrm{kg} / \mathrm{m}^{2}\right)$ & $23.7 \pm 2.3$ & $24.3 \pm 2.8$ & 0.125 & $23.8 \pm 2.6$ & $24.6 \pm 2.8$ & 0.024 \\
\hline Smoking, n (\%) & $22(44.0 \%)$ & $116(45.5 \%)$ & 0.847 & $34(34.0 \%)$ & $82(52.9 \%)$ & 0.003 \\
\hline Hypertension, n (\%) & $28(56 \%)$ & $159(62.4 \%)$ & 0.399 & $60(60.0 \%)$ & $99(63.9 \%)$ & 0.533 \\
\hline Diabetes, n (\%) & $9(18 \%)$ & $58(22.7 \%)$ & 0.459 & $22(22.0 \%)$ & $36(23.2 \%)$ & 0.820 \\
\hline Statin therapy, n (\%) & - & $110(43.1 \%)$ & - & $43(43.0 \%)$ & $67(43.2 \%)$ & 0.972 \\
\hline $\mathrm{TC}(\mathrm{mmol} / \mathrm{L})$ & $4.01 \pm 0.73$ & $4.56 \pm 0.99$ & $<0.001$ & $4.34 \pm 0.91$ & $4.70 \pm 1.01$ & 0.013 \\
\hline TG (mmol/L) & $1.09 \pm 0.32$ & $1.90 \pm 0.90$ & $<0.001$ & $1.78 \pm 0.94$ & $1.97 \pm 0.87$ & 0.025 \\
\hline LDL-C (mmol/L) & $2.48 \pm 0.60$ & $2.81 \pm 0.70$ & 0.012 & $2.68 \pm 0.69$ & $2.89 \pm 0.69$ & 0.035 \\
\hline $\mathrm{HDL}-\mathrm{C}(\mathrm{mmol} / \mathrm{L})$ & $1.13 \pm 0.18$ & $1.08 \pm 0.22$ & 0.035 & $1.11 \pm 0.21$ & $1.05 \pm 0.22$ & 0.020 \\
\hline Fasting glucose (mmol/L) & $4.95 \pm 0.93$ & $5.08 \pm 1.41$ & 0.477 & $5.08 \pm 1.45$ & $5.07 \pm 1.39$ & 0.878 \\
\hline \multicolumn{7}{|l|}{ Number of disease vessels } \\
\hline Single-vessel, n (\%) & - & - & - & - & $52(33.6 \%)$ & - \\
\hline Double-vessel, n (\%) & - & - & - & - & $40(25.8 \%)$ & - \\
\hline Triple-vessel, n (\%) & - & - & - & - & $63(40.6 \%)$ & - \\
\hline
\end{tabular}

$\mathrm{CAD}=$ coronary artery disease; $\mathrm{BMI}=$ body mass index; $\mathrm{TC}=$ total cholesterol; $\mathrm{TG}=$ triglyceride; $\mathrm{LDL}-\mathrm{C}=$ low density lipoprotein cholesterol; $\mathrm{HDL}-\mathrm{C}=$ high density lipoprotein cholesterol. 
difference was observed in age, gender, body mass index (BMI), smoking, hypertension, diabetes, or fasting glucose between two groups. In the hyperlipidemia group, when compared with CAD patients, non-CAD patients had a higher prevalence of smoking and higher levels of BMI, TC, TG, LDL-C, but lower HDL-C. No significant difference was found in age, gender, hypertension, diabetes, fasting glucose, or statin therapy between the two statuses. By coronary angiography, 52 (33.6 \%) CAD cases had single-vessel disease, 40 (25.8 \%) had doublevessel disease and 63 (40.6 \%) had triple-vessel disease.

\section{Plasma levels of miR-122 and miR-370 are increased in patients with hyperlipidemia}

Plasma levels of miR-122 and miR-370 were higher in patients with hyperlipidemia than in controls (median: 66.5 vs. $33.9, P=0.008$ for miR-122, 52.5 vs. 15.9, $P=0.001$ for miR-370, Figure 1A and 1B). Notably, statin therapy affected plasma levels of miR-122 and miR-370. Subgroup analysis revealed significantly lower plasma levels of miR-122 and miR-370 in statin-treated patients than in statin-free counterparts (median: 45.2 vs. 79.1, $P=0.012$ for miR-122, 40.0 vs. $70.9, P=0.002$ for miR-370,
Figure $1 \mathrm{C}$ and $1 \mathrm{D})$. However, plasma levels of miR-33a and miR-33b were below the detection limit in both the hyperlipidemia and control groups.

The correlations of plasma levels of miR-122 and miR-370 with blood lipid profiles

We further analyzed the correlations of plasma levels of miR-122 and miR-370 with blood lipid profiles. As shown in Table 2, the plasma levels of miR-122 and miR-370 were positively correlated with TC, TG, and LDL-C levels in both control subjects and statin-free patients with hyperlipidemia, whereas no significant correlation was observed between miRNA levels and HDL-C levels. Still, a positive correlation between plasma levels of miR-122 and miR-370 was also found. However, there was no significant association between miRNA levels and blood lipid profiles in statin-treated patients with hyperlipidemia.

\section{Increased plasma levels of miR-122 and miR-370 are} associated with the presence of CAD in patients with hyperlipidemia

In the hyperlipidemia group, CAD patients had higher plasma levels of miR-122 and miR-370 than non-CAD patients (median: 78.5 vs. $59.5, P=0.023$ for miR-122,

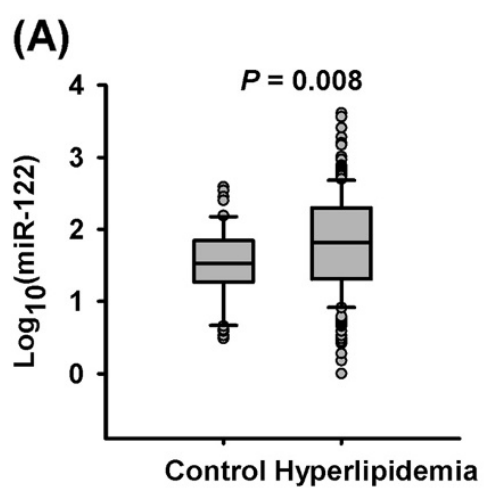

(C)

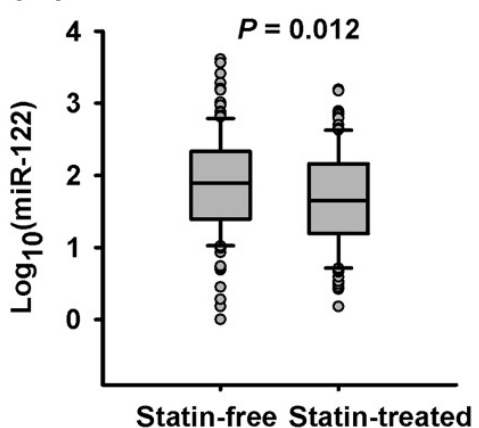

(B)

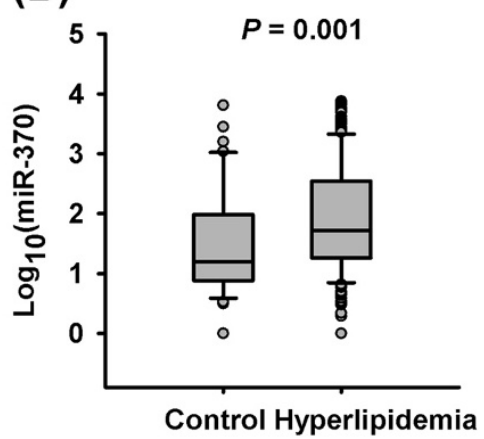

(D)

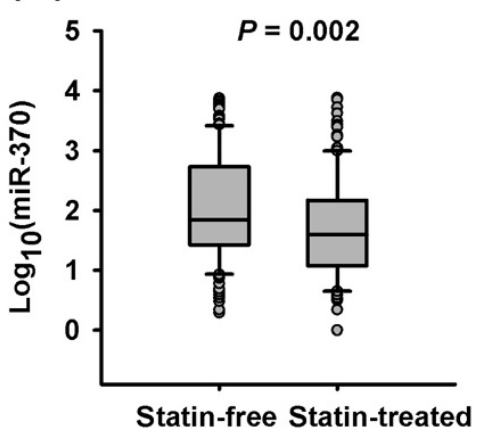

Figure 1 Plasma levels of miR-122 and miR-370 in hyperlipidemia patients and controls with normal blood lipids. Plasma levels of miR-122 (A) and miR-370 (B) are increased in hyperlipidemia patients compared with control subjects. Plasma levels of miR-122 (C) and miR-370 (D) are decreased in statin-treated patients compared with statin-free patients. 
Table 2 Spearman correlations of plasma levels of miR-122 and miR-370 with blood lipid profiles

\begin{tabular}{lllllll}
\hline & TC & TG & LDL-C & HDL-C miR-122 & miR-370 \\
\hline $\begin{array}{l}\text { Controls } \\
(\mathbf{n}=\mathbf{1 0 0})\end{array}$ & & & & & & \\
miR-122 & $0.351^{*}$ & $0.229^{*}$ & $0.352^{*}$ & -0.033 & - & $0.448^{* *}$ \\
miR-370 & $0.275^{*}$ & $0.275^{*}$ & $0.249^{*}$ & -0.003 & $0.448^{* *}$ & -
\end{tabular}

All Hyperlipidemia

$(\mathbf{n}=\mathbf{2 5 5})$

Statin-free

( $n=145)$

miR-122

miR-370

$0.322^{* *} 0.363^{* *} 0.357^{* *}-0.049-\quad 0.363^{* *}$

Statin-treated

$(n=110)$

miR-122

$\begin{array}{llllll}0.099 & 0.068 & 0.055 & -0.038 & - & 0.466^{* *} \\ 0.083 & 0.040 & 0.102 & 0.024 & 0.466^{* *} & -\end{array}$

miR-370

$0.083 \quad 0.040 \quad 0.102 \quad 0.024 \quad 0.466^{* *}$

Correlations are presented as correlation coefficients (R) and significance $(P<0.05)$ is indicated as follows: $P<0.05,{ }^{* *} P<0.001$.

$\mathrm{TC}=$ total cholesterol; $\mathrm{TG}=$ triglyceride; $\mathrm{LDL}-\mathrm{C}=$ low density lipoprotein

cholesterol; $\mathrm{HDL}-\mathrm{C}=$ high density lipoprotein cholesterol.

75.6 vs. $48.7, P=0.009$ for miR-370, Figure $2 \mathrm{~A}$ and $2 \mathrm{~B}$ ). Univariate and multivariate logistic regression analysis revealed that plasma levels of miR-122 and miR-370 were significantly associated with the presence of CAD, even after adjustment for age, gender, BMI, smoking, hypertension, diabetes, and blood lipid profiles (Table 3).

The correlations of plasma levels of miR-122 and miR-370 with the severity of CAD

CAD patients were subdivided into three subgroups (single-, double- and triple-vessel disease) according to the number of affected coronary arteries. As the number of affected vessels increased, the Gensini score significantly increased (median: 10.0 vs. 33.5 vs. 53.0, $P$ $<0.001$, Figure 3A). However, no significant difference
Table 3 Univariate analysis and multiple logistic regression analysis for the risk of CAD in patients with hyperlipidemia

\begin{tabular}{|c|c|c|c|}
\hline Models & OR & $95 \% \mathrm{Cl}$ & $P$ value \\
\hline \multicolumn{4}{|l|}{ miR-122 } \\
\hline Univariate analysis & 1.08 & $1.01-1.16$ & 0.015 \\
\hline Multiple logistic regression model $1^{a}$ & 1.08 & $1.01-1.16$ & 0.024 \\
\hline Multiple logistic regression model $2^{\mathrm{b}}$ & 1.09 & $1.01-1.17$ & 0.023 \\
\hline Multiple logistic regression model $3^{c}$ & 1.08 & $1.01-1.16$ & 0.034 \\
\hline \multicolumn{4}{|l|}{ miR-370 } \\
\hline Univariate analysis & 1.05 & $1.01-1.10$ & 0.014 \\
\hline Multiple logistic regression model $1^{a}$ & 1.04 & $1.01-1.10$ & 0.011 \\
\hline Multiple logistic regression model $2^{\mathrm{b}}$ & 1.04 & $1.01-1.09$ & 0.011 \\
\hline Multiple logistic regression model $3^{c}$ & 1.05 & $1.01-1.12$ & 0.022 \\
\hline
\end{tabular}

a The model included age, gender, BMI, and smoking.

b The model included age, gender, BMI, smoking, hypertension, and diabetes. ' The model included age, gender, BMI, smoking, hypertension, diabetes, TC, TG, LDL-C, and HDL-C.

$\mathrm{OR}=$ odds ratio; $\mathrm{Cl}=$ confidence interval.

in miR-122 and miR-370 levels was found among the three subgroups (median: 79.1 vs. 63.1 vs. $80.2, P=0.784$ for $\mathrm{miR}-122,101.2$ vs. 130.5 vs. $63.5, P=0.555$ for miR-370). Spearman correlation analysis demonstrated positive correlations of miR-122 and miR-370 levels with the severity of CAD, quantified by the Gensini score $(\mathrm{R}=0.265, P=0.040$ for $\mathrm{miR}-122, \mathrm{R}=0.247$, $P=0.014$ for miR-370, Figure $3 \mathrm{~B}$ and $3 \mathrm{C}$ ).

\section{Discussion}

Although accumulating evidence suggests that miRNAs are important regulators of lipid metabolism, little is known about the circulating levels of these lipometabolism-related miRNAs and their relationship with the presence of CAD in patients with hyperlipidemia. In the present study, we demonstrated for the first time that plasma levels of lipometabolism-related miR-122 and
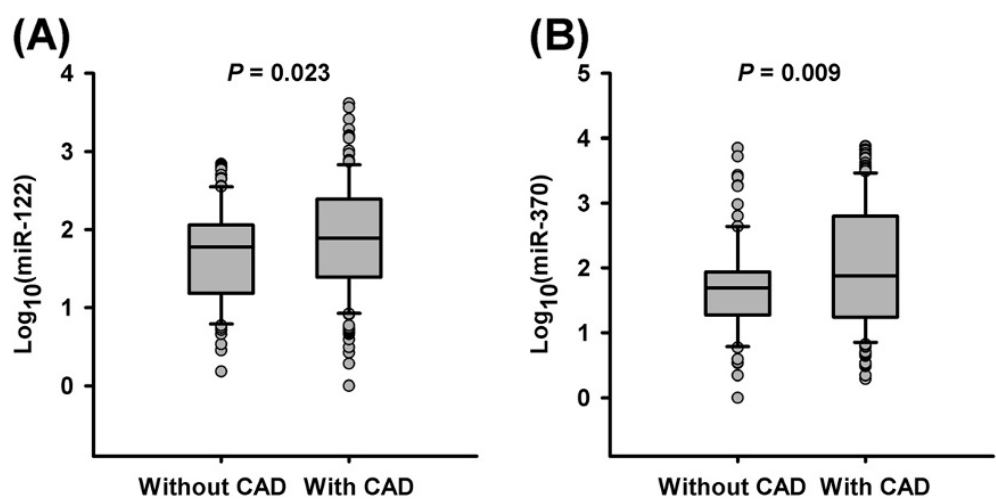

Figure 2 Plasma levels of miR-122 and miR-370 in hyperlipidemia patients with and without CAD. Plasma levels of miR-122 (A) and miR-370 (B) are increased in patients with CAD compared with those without CAD. CAD = coronary artery disease. 


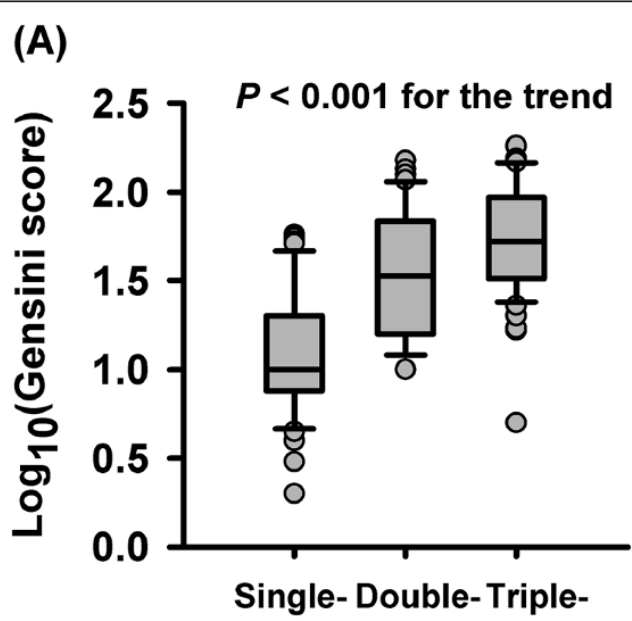

(B)

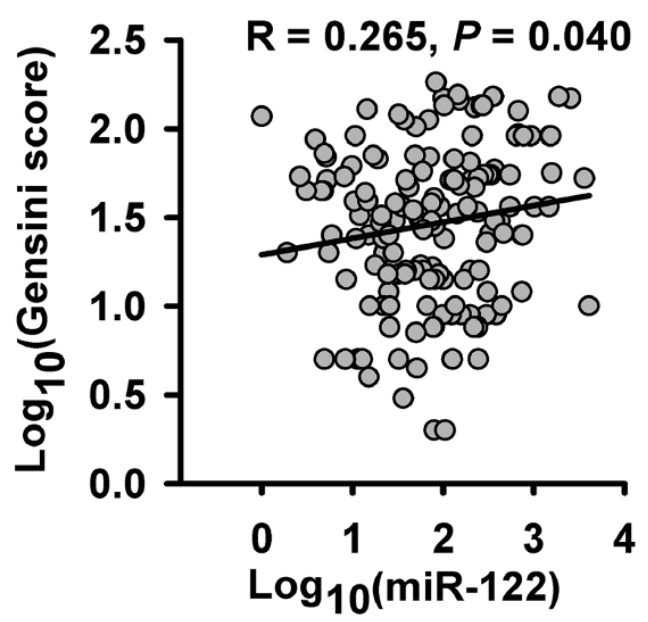

(C)

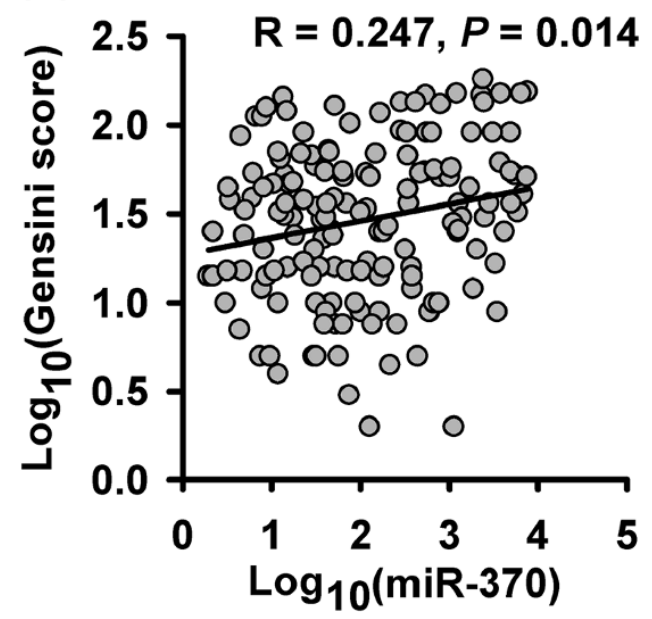

Figure 3 The correlations of plasma levels of miR-122 and miR-370 with the severity of CAD.(A) Gensini score increases as the number of affected vessels increases. Plasma levels of miR-122 (B) and miR-370 (C) are positively correlated with the Gensini score.
miR-370 were significantly increased in hyperlipidemia patients compared with controls, and the levels of miR-122 and miR-370 were positively correlated with TC, TG, and LDL-C levels in both hyperlipidemia patients and controls. Furthermore, the increased levels of miR-122 and miR-370 were associated with CAD presence, independent of other cardiovascular risk factors. In addition, miR-122 and miR-370 levels were positively correlated with the severity of CAD quantified by the Gensini score. However, both miR$33 \mathrm{a}$ and miR-33b were undetectable in plasma, indicating that miR-33a/b might not present in plasma.

It has been shown that miR-122 and miR-370 are over-expressed in the livers of hyperlipidemia animals $[7,9,22]$. The present study is the first to investigate the circulating levels of miR-122 and miR-370 in patients with hyperlipidemia. Indeed, plasma levels of miR-122 and miR-370 were also increased in patients with hyperlipidemia, and positively correlated with TC, TG, and LDL-C levels, suggesting that circulating miR-122 and miR-370 might be used as novel biomarkers for hyperlipidemia. In addition, Iliopoulos et al. [9] reported that transfection of the human hepatic cell line HepG2 with miR-370 could up-regulate the expression of miR-122. In the present study, we also found a positive correlation between plasma levels of miR-122 and miR-370, indicating that similar regulation might also exist in circulating system.

Considering the crucial role of hyperlipidemia in the onset and development of CAD, dysregulation of lipometabolism-related miRNAs might also be related to the presence of CAD. Previous studies have found that expression levels of miR-122 are increased in infarcted areas as well as in border areas after acute myocardial infarction (AMI) [23,24]. Hoekstra et al. [25] also reported that miR-370 levels were higher in peripheral blood mononuclear cells from patients with unstable angina pectoris as compared to patients with stable angina pectoris. Our observations of increased plasma levels of miR-122 and miR-370 in hyperlipidemia patients with CAD in comparison with those without CAD are consistent with these previous findings and suggest that the increased levels of miR-122 and miR-370 might be novel risk factors for CAD.

In contrast to previous findings and our results, D'Alessandra et al. [24] found that plasma levels of miR-122 were lower in AMI patients than healthy controls. Corsten's study [26] indicated that the plasma level of miR-122 was elevated in acute heart failure, while no significant change was observed in patients with AMI and myocardial diastolic dysfunction. Similarly, Wang et al. [27] found no significant difference of plasma miR-122 levels between AMI patients and healthy subjects. The different 
observations of the association between plasma miR122 level and CAD may be explained, at least in part, by the different characters of patients among different studies. Our study population is a cohort of patients with hyperlipidemia, whereas other studies focus on patients with AMI without regard to the blood lipid levels. Another possibility that may account for the observed inconsistencies across studies is that the sample sizes of other studies were smaller than our study. Nevertheless, further studies including larger and different patient cohorts are needed to fully evaluate the relationship between plasma miR-122 level and CAD.

The clinical significance of circulating lipometabolismrelated miRNAs in CAD is another aspect of great interest. To the best of our knowledge, no data has been published on the association of the circulating miRNAs with the severity of CAD. Our study demonstrated positive correlations of plasma levels of both miR-122 and miR-370 with the Gensini score, indicating that plasma levels of miR-122 and miR-370 increased as the severity of CAD increased. Thus, the potential prognostic value of these two lipometabolism-related miRNAs in CAD needs to be further evaluated. At the same time, it has been reported that miRNAs levels are reduced after statin therapy [28,29]. Our data also showed that plasma levels of miR-122 and miR-370 were lower in statin-treated patients than in statin-free subjects. Therefore, we speculate that statin therapy may also cause the reduction of circulating lipometabolism-related miRNAs. Further experimental studies are needed to explore the mechanisms by which statin therapy influences the circulating levels of lipometabolism-related miRNAs.

In conclusion, this study shows that plasma levels of miR-122 and miR-370 are increased in patients with hyperlipidemia and positively correlated with TC, TG, and LDL-C levels. Furthermore, the increased levels of miR-122 and miR-370 were associated with CAD presence. High miR-122 and miR-370 levels seemed to positively correlate with the severity of CAD.

Competing interests

The authors declare no conflict of interests.

\section{Acknowledgements}

This work was supported by a grant from the National Natural Science Foundation of China (No. 30871078).

\section{Author details \\ ${ }^{1}$ Department of Cardiology, The First Affiliated Hospital of Nanjing Medical University, Nanjing, 210029, China. ${ }^{2}$ Department of geriatrics, The Second Affiliated Hospital of Nanjing Medical University, Nanjing, 210029, China.}

\section{Authors' contributions}

Wei Gao and Lian-Sheng Wang performed the data analysis and drafted the manuscript. Hui-Wei He and Lian-Sheng Wang designed the study. Ze-Mu Wang made the graphics. Hui-Wei He, Huan Zhao and Jun Zhu collected the clinical data, which was supervised by Lian-Sheng Wang and Zhi-Jian Yang. Wei Gao, Xiao-Qing Lian and Yong-Sheng Wang performed the laboratory experiments, which was supervised and analyzed by Jian-Jun Yan and DingGuo Zhang. All authors read and approved the final manuscript.

Received: 21 March 2012 Accepted: 15 May 2012

Published: 15 May 2012

\section{References}

1. Hansson GK: Inflammation, atherosclerosis, and coronary artery disease. N Engl J Med 2005, 352:(16):1685-1695.

2. Moller DE, Kaufman KD: Metabolic syndrome: a clinical and molecular perspective. Annu Rev Med 2005, 56:45-62.

3. Bartel DP: MicroRNAs: genomics, biogenesis, mechanism, and function. Cell 2004, 116(2):281-297.

4. Fernandez-Hernando C, Suarez $Y$, Rayner K, Moore KJ: MicroRNAs in lipid metabolism. Curr Opin Lipidol 2011, 22(2):86-92.

5. Esau C, Davis S, Murray SF, Yu XX, Pandey SK, Pear M, Watts L, Booten SL, Graham M, McKay R, et al: miR-122 regulation of lipid metabolism revealed by in vivo antisense targeting. Cell Metab 2006, 3(2):87-98.

6. Krutzfeldt J, Rajewsky N, Braich R, Rajeev KG, Tuschl T, Manoharan M, Stoffel M: Silencing of microRNAs in vivo with 'antagomirs'. Nature 2005, 438 (7068):685-689.

7. Elmen J, Lindow M, Schutz S, Lawrence M, Petri A, Obad S, Lindholm M, Hedtjarn M, Hansen HF, Berger U, et al: LNA-mediated microRNA silencing in non-human primates. Nature 2008, 452(7189):896-899.

8. Lanford RE, Hildebrandt-Eriksen ES, Petri A, Persson R, Lindow M, Munk ME, Kauppinen S, Orum H: Therapeutic silencing of microRNA-122 in primates with chronic hepatitis C virus infection. Science 2010, 327(5962):198-201.

9. Iliopoulos D, Drosatos K, Hiyama Y, Goldberg IJ, Zannis VI: MicroRNA-370 controls the expression of microRNA-122 and Cpt1alpha and affects lipid metabolism. J Lipid Res 2010, 51(6):1513-1523.

10. Rayner KJ, Suarez Y, Davalos A, Parathath S, Fitzgerald ML, Tamehiro N, Fisher EA, Moore KJ, Fernandez-Hernando C: MiR-33 contributes to the regulation of cholesterol homeostasis. Science 2010, 328(5985):1570-1573.

11. Marquart TJ, Allen RM, Ory DS, Baldan A: miR-33 links SREBP-2 induction to repression of sterol transporters. Proc Natl Acad Sci U S A 2010, 107 (27):12228-12232.

12. Najafi-Shoushtari SH, Kristo F, Li Y, Shioda T, Cohen DE, Gerszten RE, Naar AM: MicroRNA-33 and the SREBP host genes cooperate to control cholesterol homeostasis. Science 2010, 328(5985):1566-1569.

13. Horie T, Ono K, Horiguchi M, Nishi H, Nakamura T, Nagao K, Kinoshita M, Kuwabara $Y$, Marusawa $H$, Iwanaga $Y$, et al: MicroRNA-33 encoded by an intron of sterol regulatory element-binding protein 2 (Srebp2) regulates HDL in vivo. Proc Natl Acad Sci U S A 2010, 107(40):17321-17326.

14. Gerin I, Clerbaux LA, Haumont O, Lanthier N, Das AK, Burant CF, Leclercq IA, MacDougald OA, Bommer GT: Expression of miR-33 from an SREBP2 intron inhibits cholesterol export and fatty acid oxidation. J Biol Chem 2010, 285(44):33652-33661.

15. Fichtlscherer S, Zeiher AM, Dimmeler S: Circulating microRNAs: biomarkers or mediators of cardiovascular diseases? Arterioscler Thromb Vasc Biol 2011, 31(11):2383-2390.

16. Creemers EE, Tijsen AJ, Pinto YM: Circulating MicroRNAs: Novel Biomarkers and Extracellular Communicators in Cardiovascular Disease?. Circ Res 2012, 110(3):483-495.

17. Jimenez-Conde J, Biffi A, Rahman R, Kanakis A, Butler C, Sonni S, Massasa E, Cloonan L, Gilson A, Capozzo K, et al: Hyperlipidemia and reduced white matter hyperintensity volume in patients with ischemic stroke. Stroke 2010, 41(3):437-442.

18. Santamore WP, Kahl FR, Kutcher MA, Negin M, Whiteman JL, Kase JP, Little WC: A microcomputer based automated, quantitative coronary angiographic analysis system. Ann Biomed Eng 1988, 16(4):367-377.

19. Gensini GG: A more meaningful scoring system for determining the severity of coronary heart disease. Am J Cardio/ 1983, 51(3):606.

20. Tang NP, Wang LS, Yang L, Zhou B, Gu HJ, Sun QM, Cong RH, Zhu HJ, Wang B: Protective effect of an endothelial lipase gene variant on coronary artery disease in a Chinese population. J Lipid Res 2008, 49 (2):369-375.

21. Mitchell PS, Parkin RK, Kroh EM, Fritz BR, Wyman SK, Pogosova-Agadjanyan EL, Peterson A, Noteboom J, O'Briant KC, Allen A, et al: Circulating microRNAs as stable blood-based markers for cancer detection. Proc Natl Acad Sci U S A 2008, 105(30):10513-10518 
22. Cirera S, Birck M, Busk PK, Fredholm M: Expression profiles of miRNA-122 and its target CAT1 in minipigs (Sus scrofa) fed a high-cholesterol diet. Comp Med 2010, 60(2):136-141

23. Dong S, Cheng Y, Yang J, Li J, Liu X, Wang X, Wang D, Krall TJ, Delphin ES, Zhang C: MicroRNA expression signature and the role of microRNA-21 in the early phase of acute myocardial infarction. J Biol Chem 2009, 284 (43):29514-29525.

24. D'Alessandra Y, Devanna P, Limana F, Straino S, Di Carlo A, Brambilla PG, Rubino M, Carena MC, Spazzafumo L, De Simone M, et al: Circulating microRNAs are new and sensitive biomarkers of myocardial infarction. Eur Heart J 2010, 31(22):2765-2773.

25. Hoekstra M, van der Lans CA, Halvorsen B, Gullestad L, Kuiper J, Aukrust P, van Berkel TJ, Biessen EA: The peripheral blood mononuclear cell microRNA signature of coronary artery disease. Biochem Biophys Res Commun 2010, 394(3):792-797.

26. Corsten MF, Dennert R, Jochems $S$, Kuznetsova T, Devaux $Y$, Hofstra L, Wagner DR, Staessen JA, Heymans S, Schroen B: Circulating MicroRNA208b and MicroRNA-499 Reflect Myocardial Damage in Cardiovascular Disease. Circ Cardiovasc Genet 2010, 3(6):499-506.

27. Wang GK, Zhu JQ, Zhang JT, Li Q, Li Y, He J, Qin YW, Jing Q: Circulating microRNA: a novel potential biomarker for early diagnosis of acute myocardial infarction in humans. Eur Heart J 2010, 31(6):659-666.

28. Zhang Q, Kandic I, Kutryk MJ: Dysregulation of angiogenesis-related microRNAs in endothelial progenitor cells from patients with coronary artery disease. Biochem Biophys Res Commun 2011, 405(1):42-46.

29. Minami Y, Satoh M, Maesawa C, Takahashi Y, Tabuchi T, Itoh T, Nakamura M: Effect of atorvastatin on microRNA 221 / 222 expression in endothelial progenitor cells obtained from patients with coronary artery disease. Eur J Clin Invest 2009, 39(5):359-367.

doi:10.1186/1476-511X-11-55

Cite this article as: Gao et al:: Plasma levels of lipometabolism-related miR-122 and miR-370 are increased in patients with hyperlipidemia and associated with coronary artery disease. Lipids in Health and Disease 2012 $11: 55$

\section{Submit your next manuscript to BioMed Central and take full advantage of:}

- Convenient online submission

- Thorough peer review

- No space constraints or color figure charges

- Immediate publication on acceptance

- Inclusion in PubMed, CAS, Scopus and Google Scholar

- Research which is freely available for redistribution 